\title{
Clinical Outcomes with a Novel Extended Depth of Focus Presbyopia-Correcting Intraocular Lens: Pilot Study
}

This article was published in the following Dove Press journal: Clinical Ophthalmology

\author{
María T Iradier' \\ Verónica Cruz' \\ Naty Gentile' \\ Priscila Cedano' \\ David P Piñero $\mathbb{D}^{2}$ \\ 'Iradier Eye Clinic, Madrid, Spain; \\ ${ }^{2}$ Department of Optics, Pharmacology \\ and Anatomy, University of Alicante, \\ Alicante, Spain
}

Purpose: To evaluate the clinical outcomes of cataract surgery with implantation of a novel model of extended depth of focus (EDOF) intraocular lens (IOL).

Methods: Pilot case series enrolling a total of 27 eyes of 16 patients (49 to 84 years) undergoing uncomplicated phacoemulsification cataract surgery with implantation of the EDOF IOL Synthesis PLUS (Cutting Edge, Montpellier, France). Near (UNVA, uncorrected near visual acuity; DCNVA, distance-corrected near visual acuity) and distance visual acuity (uncorrected and corrected distance visual acuity, UDVA and CDVA), monocular defocus curve and refractive outcomes were evaluated during a 3-month follow-up.

Results: Mean postoperative UDVA, UNVA and DCNVA were $0.11 \pm 0.17,0.14 \pm 0.22$ and 0.37 $\pm 0.36 \log$ MAR, respectively. A total of $84.6 \%, 91.7 \%$, and $96.3 \%$ of eyes achieved postoperative UDVA, UNVA and CDVA of $0.20 \log$ MAR or better. A total of $78.6 \%$ of eyes achieved postoperative DCNVA of $0.30 \log$ MAR or better. Mean postoperative spherical equivalent was $-0.76 \pm 0.53 \mathrm{D}$. The distance-corrected visual acuity was maintained on average over a value of $0.30 \log$ MAR for the range of defocus levels between +1.00 and $-1.50 \mathrm{D}$.

Conclusion: The implantation of the Synthesis plus EDOF IOL after cataract surgery seems to provide functional levels of distance, intermediate and near visual acuity. The near visual performance with this new IOL might be significantly enhanced using a micro-monovision approach. The results of this pilot study should be confirmed in future clinical trials.

Keywords: presbyopia, cataract surgery, presbyopia-correcting IOL, extended depth of focus IOL, defocus curve

\section{Introduction}

A fast evolution of presbyopia-correcting intraocular lenses (IOL) has occurred in last years, with the development of new models and designs. ${ }^{1,2}$ Extended depth of focus (EDOF) IOLs is one of these options for presbyopia correction that has experienced a prolific development in recent years. ${ }^{3}$ The basic principle behind these IOLs is to create a single elongated focal point to enhance the depth of focus or range of vision, allowing a functional vision at different distances in presbyopia. ${ }^{4}$ This effect can be achieved by inducing controlled levels of spherical aberration, ${ }^{5}$ combining refractive and diffractive surfaces to optimize achromatic and chromatic aberrations $^{6,7}$ or using a pinhole design. ${ }^{7}$

Several studies have been conducted to this date to evaluate the outcomes with different commercially available EDOF IOLs, confirming the presence of some differences in the clinical performance of some IOL models and consequently in the
Correspondence: David P Piñero

Department of Optics, Pharmacology and Anatomy, University of Alicante, Crta San Vicente del Raspeig s/n, San Vicente del Raspeig (Alicante), 03690, Spain

Email david.pinyero@ua.es 
visual rehabilitation provided. ${ }^{1,2,4,7-21}$ A common finding with all types of EDOF IOLs is an effective visual restoration at intermediate vision, but the level of near vision achieved varies significantly among IOL models. ${ }^{1,2,4,7-21}$ A novel recent EDOF approach has been developed for presbyopia correction based on the combination of primary and secondary spherical aberrations of opposite signs with a transition zone to a peripheral monofocal area (Synthesis PLUS, Cutting Edge, Montpellier, France). The theoretical aim of this new design is to deliver continuous high-contrast vision from distance to intermediate vision, while maintaining a functional level of near vision and preserving the ocular optical quality within an acceptable range to avoid the induction of light disturbances, such as halos, glare or starbursts. However, to this date, there are no scientific reports on the clinical outcomes obtained with this new model of EDOF IOL. The aim of the current clinical study was to evaluate the clinical outcomes in terms of distance and near visual acuities, refractive predictability and binocular defocus curve in a sample of eyes undergoing cataract surgery with implantation of the Synthesis EDOF IOL.

\section{Methods}

\section{Patients}

This pilot case series enrolled a total of 27 eyes of 16 patients undergoing uncomplicated phacoemulsification cataract surgery with implantation of the EDOF IOL Synthesis PLUS (Cutting Edge, Montpellier, France). All patients were adequately informed about the study in the preoperative visit and signed a consent form in accordance with the tenets of the Declaration of Helsinki. The study was approved by the Iradier clinic ethics committee. Inclusion criteria were cataract or presbyopic/prepresbyopic patients suitable for refractive lens exchange seeking for spectacle independence and predicted postoperative astigmatism equal or less than 1.00 D. Exclusion criteria were previous ocular surgery, active or systemic ocular pathology, irregular corneal astigmatism, abnormal iris, and antecedents of ocular conditions such as glaucoma, uveitis or retinal problems.

\section{Clinical Protocol}

A complete preoperative ophthalmological examination was performed in all patients including manifest refraction, measurement of monocular uncorrected (UDVA) and corrected distance visual acuity (CDVA), corneal topography and pachymetry with the Pentacam HR system (OCULUS Optikgeräte GmbH, Wetzlar, Germany), slitlamp examination, Goldmann applanation tonometry, optical biometry and keratometry (IOL Master 700, Carl Zeiss Meditec, Jena, Germany), infrared pupillometry and indirect ophthalmoscopy.

Postoperatively, besides the day after surgery, all patients were evaluated at 1 month and 3 months postoperatively. The examination the day after surgery included measurement of monocular UDVA and uncorrected near visual acuity (UNVA) $(40 \mathrm{~cm})$, tonometry and biomicroscopic examination of the integrity of the anterior segment. At 3 months after surgery, the examination performed consisted of the following tests: measurement of monocular UDVA and UNVA, manifest refraction, measurement of monocular CDVA and distance-corrected near visual acuity (DCNVA), slit-lamp biomicroscopic examination, and monocular evaluation of the defocus curve to evaluate the range of functional function. The defocus curve was measured using Snellen charts at $5 \mathrm{~m}$, while the patient was wearing the spherocylindrical refraction providing the best distance visual acuity. Different levels of defocus were introduced in $0.5 \mathrm{D}$ steps from $+0.50 \mathrm{D}$ to $-3.50 \mathrm{D}$, and visual acuity values were recorded. All these data were then represented in a Cartesian graphic display, with the $\mathrm{x}$-axis showing the levels of defocus and the y-axis the visual acuity achieved.

\section{Surgery}

All surgeries were performed by the same experienced surgeon (MTI) using a standard technique of sutureless microincision phacoemulsification. First, anaesthesia and mydriatic drops were instilled and the surgery was initiated with the creation of the corneal incision at the temporal area. After this, the capsulorrhexis was created manually and the phacoemulsification was performed. Finally, the IOL was inserted into the capsular bag through the main incision using the Medicel Accuject Pro $1.6 \mathrm{~mm}$ injector (Medicel AG, Thal, Switzerland). A combination of topical antibiotic and steroid was prescribed to be applied postoperatively four times daily for one week.

\section{Intraocular Lens}

The Synthesis PLUS IOL is a one-piece EDOF IOL with a $6.0-\mathrm{mm}$ aspheric optic, and a variable overall length depending on its power $(11.0 \mathrm{~mm}$ : 0 to $15.0 \mathrm{D}, 10.7 \mathrm{~mm}$ : 15.25 to $22.0 \mathrm{D}$, and $10.5 \mathrm{~mm}: 22.25$ to $32.0 \mathrm{D}$ ). It has a 4 point-fixation haptics and a continuous $360^{\circ}$ posterior 
square optic edge, with a shift aimed at promoting capsular bag adhesion (angulation $0^{\circ}$ ). It is made of a hydrophilic acrylic material with a refractive index of 1.459 . The IOL has a continuous area with an EDOF central zone, a patented transition zone and monofocal optical periphery, generating a combination of primary and secondary spherical aberrations of opposite signs promoting an increase of the depth of field. The company labelled A-constant for this IOL is 118.0. The IOL power calculations were conducted in this study using the Barrett Universal II (BU-II) formula and considering a target refraction of emmetropia in the dominant eye and minimal myopic refractive error (around $-0.50 \mathrm{D})$ in the non-dominant eye.

\section{Data Analysis}

The SPSS software package (SPSS Version 20.0; IBM Corporation, Armonk, NY, USA) was used to perform the analysis of data obtained in this study. Normality of data samples was evaluated by means of the KolmogorovSmirnov test. When parametric analysis was possible, the Student's $t$-test for paired data was used for the analysis of differences between preoperative and postoperative visits, whereas the Wilcoxon ranked sum test was used for such purpose when the data samples were not normally distributed. A p-value below 0.05 was considered as a criterion of statistical significance.

\section{Results}

A total of 27 eyes from 16 patients ranging in age from 49 to 84 years were included in the study. Monocular implantation of the IOL was performed in 5 patients $(31.3 \%)$. In the 11 patients having a bilateral implantation of the IOL, a symmetric target refraction was planned in 7 patients (43.8\%), whereas a micromonovision approach was planned in 4 patients $(25 \%$ ) (4 eyes with a myopic target of $-0.50 \mathrm{D}$ or more). The sample was comprised of 10 females (62.5\%) and 6 males (37.5\%). Mean axial length, anterior chamber depth and keratometry were $23.91 \mathrm{~mm}$ (standard deviation, SD: 1.13; median: 23.74; range: 22.03 to $26.25 \mathrm{~mm}$ ), $2.94 \mathrm{~mm}$ (SD: 0.34; median: 3.00; range: 2.14 to $3.37 \mathrm{~mm}$ ), and $42.19 \mathrm{D}$ (SD: 2.01; median: 42.25; range: 39.00 to $46.15 \mathrm{D})$, respectively. Mean IOL power implanted was 21.78 D (standard deviation, SD: 2.96; median: 21.00 ; range: 17.50 to $28.50 \mathrm{D}$ ).

Table 1 summarizes the preoperative and 3-month postoperative monocular visual and refractive data. Changes in manifest refraction did not reach statistical significance $(\mathrm{p} \geq 0.249$ ) mainly due to the large variability of the preoperative data. However, a statistically significant improvement was observed in UDVA and CDVA $(\mathrm{p}=0.001)$. Likewise, a statistically significant improvement was found in UNVA $(\mathrm{p}<0.001)$. A total of $84.6 \%(22 / 26), 91.7 \%(22 / 24)$, and $96.3 \%(26 / 27)$ of eyes achieved UDVA, UNVA and CDVA

Table I Summary of the Preoperative and Postoperative Monocular Visual and Refractive Data of the Analyzed Sample

\begin{tabular}{|c|c|c|c|}
\hline Mean (SD) & \multirow[t]{2}{*}{ Preoperative } & \multirow[t]{2}{*}{3 Months Postop } & \multirow[t]{2}{*}{ p-value } \\
\hline Median (Range) & & & \\
\hline LogMAR UDVA & $\begin{array}{c}0.69(0.65) \\
0.45(0.09 \text { to } 2.00)\end{array}$ & $\begin{array}{c}0.11(0.17) \\
0.06(-0.07 \text { to } 0.70)\end{array}$ & 0.001 \\
\hline LogMAR CDVA & $\begin{array}{c}0.20(0.24) \\
0.12(0.00 \text { to } 0.82)\end{array}$ & $\begin{array}{c}0.04(0.1 \mathrm{I}) \\
0.00(-0.07 \text { to } 0.38)\end{array}$ & 0.001 \\
\hline Sphere (D) & $\begin{array}{c}0.10(3.46) \\
0.63(-9.75 \text { to } 5.50)\end{array}$ & $\begin{array}{c}-0.49(0.5 \mathrm{I}) \\
-0.50(-\mathrm{I} .50 \text { to } 0.50)\end{array}$ & 0.249 \\
\hline Cylinder (D) & $\begin{array}{c}-0.70(0.70) \\
-0.50(-2.50 \text { to } 0.00)\end{array}$ & $\begin{array}{c}-0.53(0.45) \\
-0.50(-1.50 \text { to } 0.00)\end{array}$ & 0.732 \\
\hline SE (D) & $\begin{array}{c}-0.25(3.64) \\
0.50(-11.00 \text { to } 4.88)\end{array}$ & $\begin{array}{c}-0.76(0.53) \\
-0.88(-1.62 \text { to } 0.12)\end{array}$ & 0.372 \\
\hline LogMAR UNVA & $\begin{array}{c}0.99(0.66) \\
1.00(0.10 \text { to } 2.00)\end{array}$ & $\begin{array}{c}0.14(0.22) \\
0.10(0.00 \text { to } 1.00)\end{array}$ & $<0.001$ \\
\hline LogMAR DCNVA & - & $\begin{array}{c}0.37(0.36) \\
0.22(0.00 \text { to } 1.00)\end{array}$ & - \\
\hline
\end{tabular}

Abbreviations: UDVA, uncorrected distance visual acuity; CDVA, corrected distance visual acuity; UNVA, uncorrected near visual acuity; CNVA, corrected near visual acuity; DCNVA, distance-corrected near visual acuity; SE, spherical equivalent; D, diopters. 
of $0.20 \log$ MAR or better at 3 months after surgery (Figure 1). A total of $78.6 \%$ of eyes achieved postoperative DCNVA of $0.30 \operatorname{logMAR}$ or better (Figure 1). A total of $85.2 \%(23 / 27)$, $70.4 \%$ (19/27) and 74.1\% (20/27) of eyes had a postoperative sphere, cylinder, and spherical equivalent within $\pm 1.00 \mathrm{D}$, respectively (Figure 2). In addition, all eyes had a spherical equivalent within $\pm 0.75 \mathrm{D}$ of the target refraction.

Figure 3 shows the mean defocus curve measured at 3 months after surgery in all cases enrolled in the study. As shown, the visual acuity was maintained on average over a value of $0.30 \operatorname{logMAR}$ for the range of defocus levels between +1.00 and $-1.50 \mathrm{D}$. The standard deviation of visual acuity measures increased as the more negative was the defocus level (0 D, SD: 0.07; -1 D, SD: 0.12; -2 D, SD: $0.28 ;-3$ D, SD: 0.35) (Figure 3). Mean change in logMAR visual acuity from 0 to $1.5 \mathrm{D}$ defocus levels was 0.27 (SD: 0.18 ; median: 0.26 ; range: -0.08 to 0.60 ).

No complications were detected and recorded during the follow-up.

\section{Discussion}

The clinical performance of a novel EDOF IOL based on the controlled induction of spherical aberration to expand the depth of focus has been evaluated in this case series. This is the first clinical experience with this new EDOF IOL reported to this date. The results obtained suggest that an enlargement of the depth of focus is induced with the implantation of this novel IOL, as the measurements of the defocus curve obtained show relatively functional levels of near and intermediate visual acuity while maintaining good levels of distance vision. Specifically, in the current series, mean 3-month postoperative monocular UDVA and UNVA values of $0.11 \pm 0.17$ and $0.14 \pm 0.22 \log$ MAR were obtained, respectively. This finding is consistent with the results of other studies evaluating the performance of other types of EDOF IOLs, ${ }^{7,8,13,17-19}$ although it should be noted that 4 micro-monovision cases were included in our sample. Giers et $\mathrm{al}^{18}$ evaluated an EDOF IOL also based on induction of controlled levels of spherical aberration (MiniWell Ready), obtaining median monocular UDVA and UNVA values of 0.13 and $0.14 \log$ MAR, respectively, at 2-4 months after its implantation. Grabner et $\mathrm{al}^{7}$ reported in patients implanted monocularly with the IC-8 IOL (pinhole design) mean 1-month postoperative UDVA and UNVA values of $0.06 \pm 0.08$ and $0.11 \pm 0.15 \log$ MAR, respectively. Therefore, the EDOF IOL evaluated can

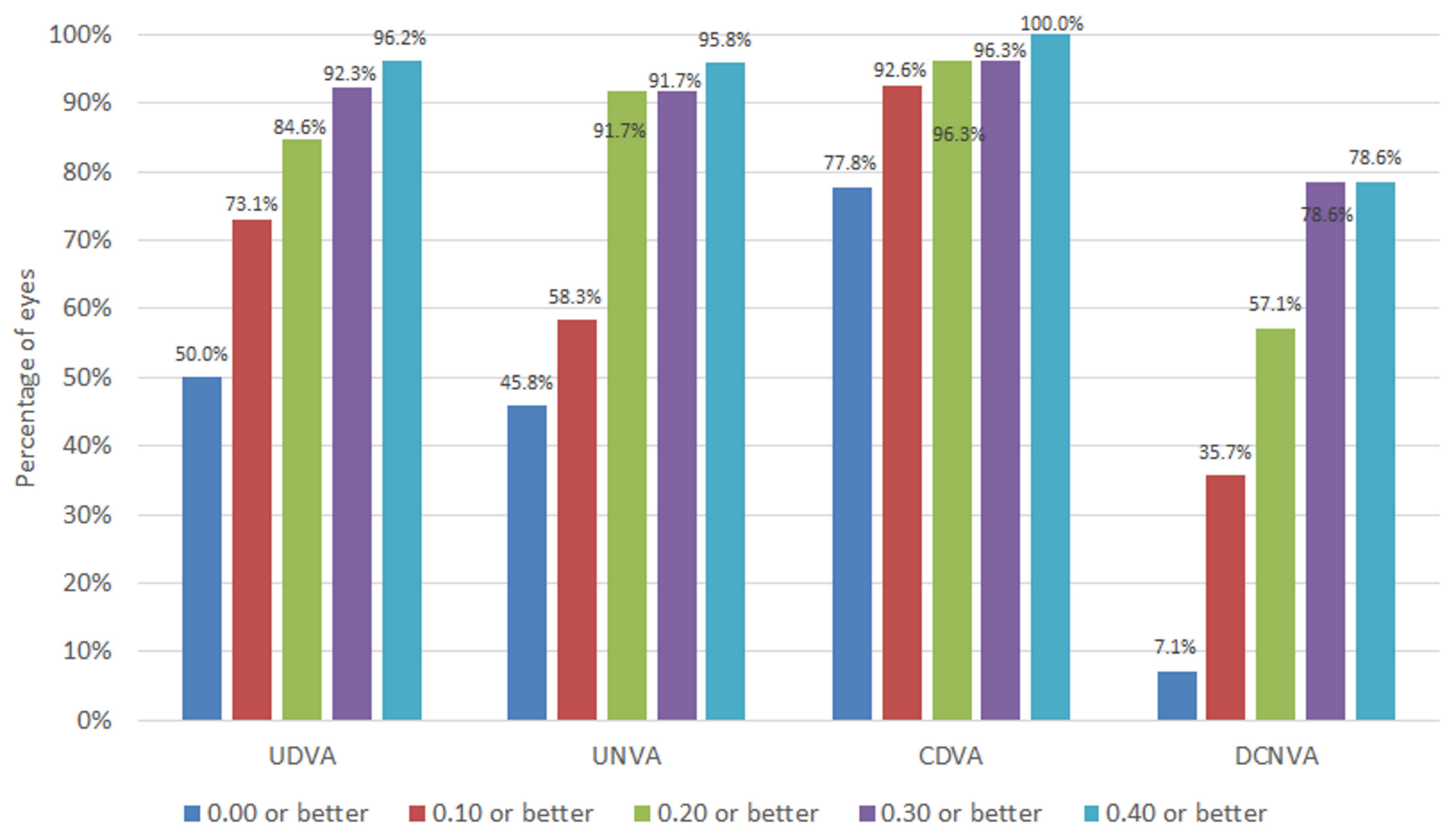

Figure I Distribution of 3-month postoperative monocular visual acuity data in the analysed sample.

Abbreviations: UDVA, uncorrected distance visual acuity; CDVA, corrected distance visual acuity; UNVA, uncorrected near visual acuity; DCNVA, distance-corrected near visual acuity. 


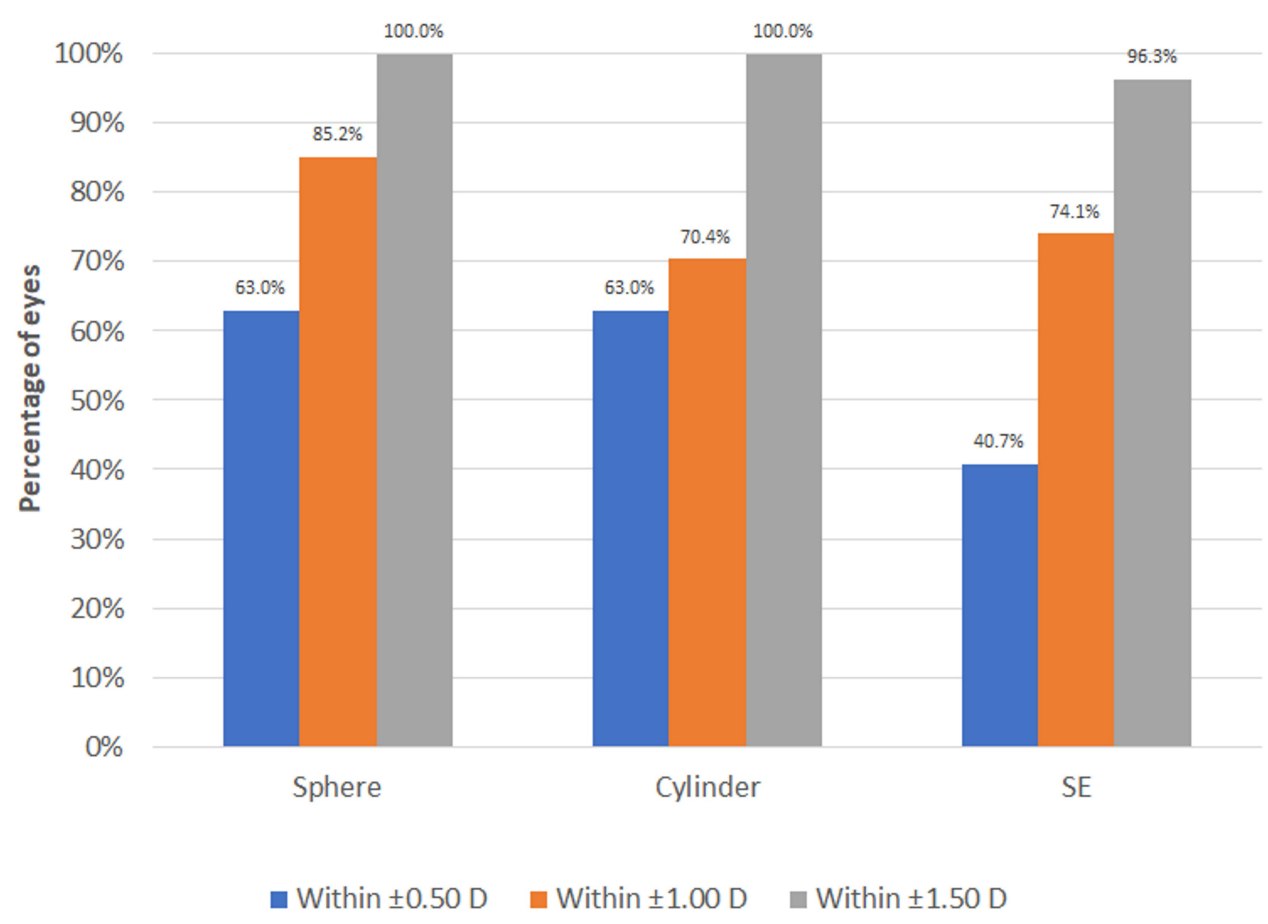

Figure 2 Distribution of 3-month postoperative refractive data in the analysed sample. Abbreviation: SE, spherical equivalent.

provide an intermediate and near visual performance compatible with that provided by other currently available EDOF IOL models.

The near visual performance with EDOF IOLs may be enhanced with a minimal residual myopic error in the nondominant eye or in both eyes. ${ }^{10,14,22}$ This approach has been used in our series, with a mean postoperative spherical equivalent of $-0.76 \pm 0.53 \mathrm{D}$ due to the inclusion of 4 micro-monovision cases. This explains the difference between monocular postoperative UNVA $(0.11 \pm 0.15$ $\log$ MAR $)$ and DCNVA $(0.37 \pm 0.36 \log$ MAR $)$, with values of this last visual parameter consistent with those reported for other refractive and diffractive EDOF IOLs. ${ }^{8,9,11,13,16,20}$ Mean DCNVA values of $0.32 \pm 0.19$ and $0.39 \pm 0.21 \operatorname{logMAR}$ were reported by Reinhard et $\mathrm{al}^{9}$ at 6 months after implantation of the diffractive IOLs AT LARA 829MP and Tecnis Symfony. Savini et $\mathrm{al}^{20}$ obtained a mean DCNVA of $0.35 \pm 0.14 \log$ MAR in

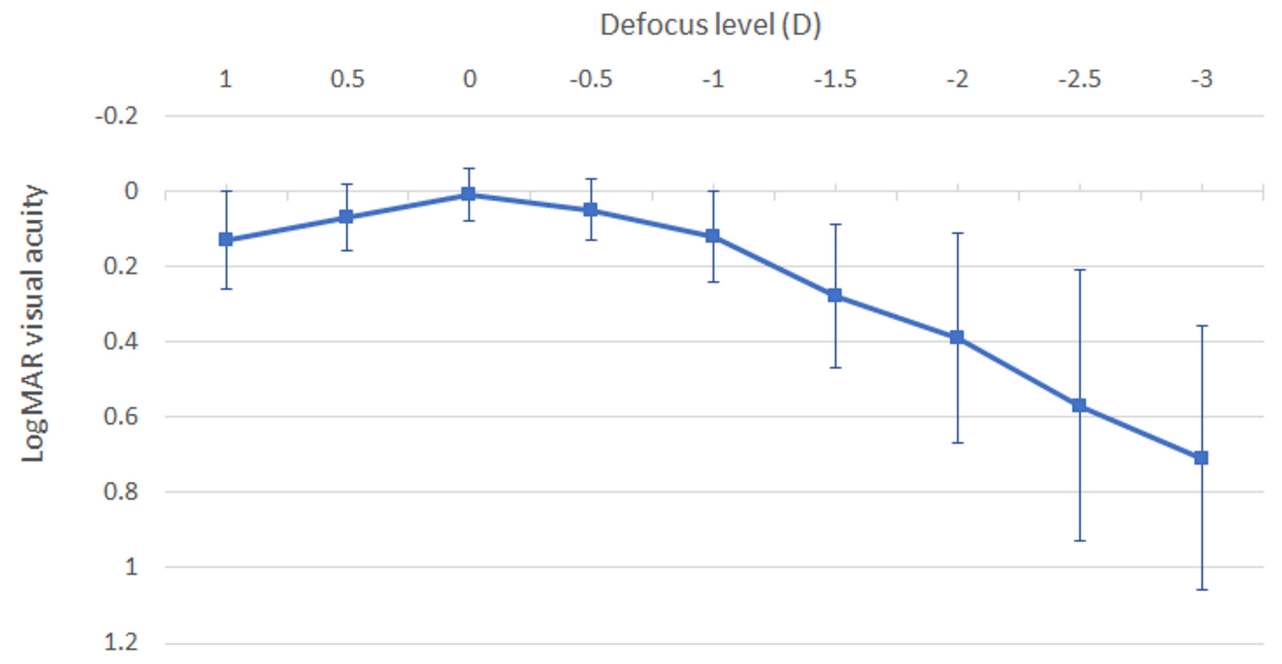

Figure 3 Mean monocular defocus curve obtained at 3 months after surgery in the analysed sample. 
a group of 20 patients bilaterally implanted with the refractive EDOF IOL MiniWell. In the current series, the use of a micro-monovision or bilateral residual myopic approach with the EDOF IOL evaluated induces a significant enhancement of the near visual acuity performance while preserving good levels of intermediate and distance vision.

Mean corrected distance visual acuities of $0.12 \pm 0.12$ and $0.28 \pm 0.19 \log$ MAR were obtained for the defocus levels of -1 and $-1.5 \mathrm{D}$ of the defocus curve. This confirms that the EDOF IOL evaluated can provide functional levels of intermediate vision, with outcomes comparable to those reported by previous authors evaluating other EDOF IOLs. 7,13,16,17,20 Savini et $\mathrm{al}^{16}$ demonstrated that the refractive EDOF IOL Mini Well increased the depth of focus through 2.0 D of defocus, with the best performance at 1.0 and $1.5 \mathrm{D}$ of defocus. This same research group reported in another study evaluating the same refractive EDOF IOL mean postoperative distancecorrected visual acuity values of $0.08 \pm 0.09$ and $0.15 \pm 0.11$ $\operatorname{logMAR}$ for the defocus of -1.0 and $-1.5 \mathrm{D}$, respectively. ${ }^{20}$ With the pinhole-based EDOF IOL IC-8, Grabner et al $^{7}$ found that $100 \%$ of eyes maintained a 12 -month postoperative visual acuity of 20/40 or better over a range of defocus from +0.50 to $-1.50 \mathrm{D}$. In the current series, the corrected distance visual acuity was maintained on average over a value of 0.30 logMAR for the range of defocus levels between +1.00 and -1.50 D. Likewise, a total of $78.6 \%$ of eyes achieved postoperative DCNVA of $0.30 \log$ MAR or better.

The present study has several limitations that should be acknowledged. First, the sample size is limited but it should be considered that this study was planned as a pilot research to evaluate the clinical performance of the new EDOF IOL evaluated. At this moment, a larger sample is being recruited to confirm in the future if these preliminary outcomes are corroborated in the short- and long-term follow-up. Second, only visual acuity and refractive data have been reported and therefore conclusions about the impact of the implant of this IOL on optical quality cannot be extracted. Future studies should be conducted including the analysis of changes in contrast sensitivity, visual symptoms using a validated questionnaire and in ocular high order aberrations. Third, the current study is not comparative and the superiority of the performance of this EDOF IOL over a conventional monofocal IOL cannot be confirmed. This is an issue that should be investigated further in future comparative studies. Finally, the lack of a standardized approach in the refractive target planning created an inhomogeneous group of patients making some of the data (especially UDVA and UNVA) difficult to interpret.

In conclusion, the implantation of the Synthesis plus EDOF IOL after cataract surgery seems to provide functional levels of distance, intermediate and near visual acuity. The near visual acuity with this new IOL can be enhanced using a micro-monovision or bilateral residual myopic approach, targeting some minimal level of residual myopia. More research is still needed to evaluate the impact of this EDOF IOL on visual quality and its advantage over other presbyopia-correcting options that are currently available. Furthermore, the results of this pilot study should be confirmed in future comparative clinical trials.

\section{Acknowledgment}

We would like to acknowledge Yvonne Hernández for her participation in this research, by assisting in its organization.

\section{Disclosure}

The authors have no proprietary or commercial interest in the medical devices that are involved in this manuscript. The authors report no conflicts of interest in this work.

\section{References}

1. Böhm M, Petermann K, Hemkeppler E, Kohnen T. Defocus curves of 4 presbyopia-correcting IOL designs: diffractive panfocal, diffractive trifocal, segmental refractive, and extended-depth-of-focus. $J$ Cataract Refract Surg. 2019;45:1625-1636. doi:10.1016/j.jcrs.20 19.07.014

2. Sudhir RR, Dey A, Bhattacharrya S, Bahulayan A. AcrySof IQ PanOptix intraocular lens versus extended depth of focus intraocular lens and trifocal intraocular lens: a clinical overview. Asia Pac J Ophthalmol (Phila). 2019;8:335-349. doi:10.1097/APO.000000000 0000253

3. Liu J, Dong Y, Wang Y. Efficacy and safety of extended depth of focus intraocular lenses in cataract surgery: a systematic review and meta-analysis. BMC Ophthalmol. 2019;19:198. doi:10.1186/s12886019-1204-0

4. Palomino-Bautista C, Sánchez-Jean R, Carmona-González D, Piñero DP, Molina-Martín A. Subjective and objective depth of field measures in pseudophakic eyes: comparison between extended depth of focus, trifocal and bifocal intraocular lenses. Int Ophthalmol. 2020;40:351-359. doi:10.1007/s10792-019-01186-6

5. Camps VJ, Tolosa A, Piñero DP, de Fez D, Caballero MT, Miret JJ. In vitro aberrometric assessment of a multifocal intraocular lens and two extended depth of focus IOLs. $J$ Ophthalmol. 2017;2017:70 95734. doi:10.1155/2017/7095734

6. Chae SH, Son HS, Khoramnia R, Lee KH, Choi CY. Laboratory evaluation of the optical properties of two extended-depth-of-focus intraocular lenses. BMC Ophthalmol. 2020;20:53. doi:10.1186/ s12886-020-1332-6

7. Grabner G, Ang RE, Vilupuru S. The small-aperture IC-8 intraocular lens: a new concept for added depth of focus in cataract patients. $\mathrm{Am}$ J Ophthalmol. 2015;160:1176-1184. doi:10.1016/j.ajo.2015.08.017 
8. Pedrotti E, Chierego C, Talli PM, et al. Extended depth of focus versus monofocal IOLs: objective and subjective visual outcomes. J Refract Surg. 2020;36:214-222. doi:10.3928/1081597X-20200212-01

9. Reinhard T, Maier P, Böhringer D, et al. Comparison of two extended depth of focus intraocular lenses with a monofocal lens: a multi-centre randomised trial. Graefes Arch Clin Exp Ophthalmol. 2020. doi:10.1007/s00417-020-04868-5

10. Schojai M, Schultz T, Jerke C, Böcker J, Dick HB. Prospective randomized comparative trial: visual performance comparison of two enhanced depth of focus IOLs - symfony and IC-8. J Cataract Refract Surg. 2020. doi:10.1097/j.jcrs.0000000000000068

11. Ozulken K, Kiziltoprak H, Yuksel E, Mumcuoğlu T. A comparative evaluation of diffractive trifocal and new refractive/extended depth of focus intraocular lenses for refractive lens exchange. Curr Eye Res. 2020;1-7. doi:10.1080/02713683.2020.1833347

12. Sinha R, Sahay P, Saxena R, Kalra N, Gupta V, Titiyal JS. Visual outcomes of binocular implantation of a new extended depth of focus intraocular lens. Indian $J$ Ophthalmol. 2020;68:2111-2116. doi:10.4103/ijo.IJO_2139_19

13. Auffarth GU, Moraru O, Munteanu M, et al. European, multicenter, prospective, non-comparative clinical evaluation of an extended depth of focus intraocular lens. J Refract Surg. 2020;36:426-434. doi:10.3928/1081597X-20200603-01

14. Schojai M, Schultz T, Jerke C, Böcker J, Dick HB. Visual performance comparison of 2 extended depth-of-focus intraocular lenses. $J$ Cataract Refract Surg. 2020;46:388-393. doi:10.1097/j. jcrs.0000000000000068

15. Son HS, Khoramnia R, Yildirim TM, Baur I, Labuz G, Auffarth GU. Functional outcomes and reading performance after combined implantation of a small-aperture lens and a segmental refractive bifocal lens. J Refract Surg. 2019;35:551-558. doi:10.3928/ 1081597X-20190806-02
16. Savini G, Balducci N, Carbonara C, et al. Functional assessment of a new extended depth-of-focus intraocular lens. Eye (Lond). 2019;33:404-410. doi:10.1038/s41433-018-0221-1

17. Bellucci R, Cargnoni M, Bellucci C. Clinical and aberrometric evaluation of a new extended depth-of-focus intraocular lens based on spherical aberration. J Cataract Refract Surg. 2019;45:919-926. doi:10.1016/j.jcrs.2019.02.023

18. Giers BC, Khoramnia R, Varadi D, et al. Functional results and photic phenomena with new extended-depth-of-focus intraocular lens. $B M C$ Ophthalmol. 2019;19:197. doi:10.1186/s12886-019-1201-3

19. Nivean M, Nivean PD, Reddy JK, et al. Performance of a new-generation extended depth of focus intraocular lens-a prospective comparative study. Asia Pac J Ophthalmol (Phila). 2019;8:285-289. doi:10.1097/APO.0000000000000245

20. Savini G, Schiano-Lomoriello D, Balducci N, Barboni P. Visual performance of a new extended depth-of-focus intraocular lens compared to a distance-dominant diffractive multifocal intraocular lens. J Refract Surg. 2018;34:228-235. doi:10.3928/1081597X-20180125-01

21. Cochener B, Boutillier G, Lamard M, Auberger-Zagnoli C. A comparative evaluation of a new generation of diffractive trifocal and extended depth of focus intraocular lenses. J Refract Surg. 2018;34:507-514. doi:10.3928/1081597X-20180530-02

22. Cochener B. Influence of the level of monovision on visual outcome with an extended range of vision intraocular lens. Clin Ophthalmol. 2018;12:2305-2312. doi:10.2147/OPTH.S184712
Clinical Ophthalmology

\section{Publish your work in this journal}

Clinical Ophthalmology is an international, peer-reviewed journal covering all subspecialties within ophthalmology. Key topics include: Optometry; Visual science; Pharmacology and drug therapy in eye diseases; Basic Sciences; Primary and Secondary eye care; Patient Safety and Quality of Care Improvements. This journal is indexed on PubMed

\section{Dovepress}

Central and CAS, and is the official journal of The Society of Clinical Ophthalmology (SCO). The manuscript management system is completely online and includes a very quick and fair peer-review system, which is all easy to use. Visit http://www.dovepress.com/ testimonials.php to read real quotes from published authors. 